\title{
Echocardiographic findings in left ventricular to right atrial shunts
}

\author{
PETER MILLS, LAMBERT MCLAURIN, CLAUDE SMITH, GORDON MURRAY, \\ AND ERNEST CRAIGE
}

From the Departments of Cardiology and Cardiothoracic Surgery, University of North Carolina, School of Medicine, Chapel Hill, N.C., U.S.A.

The echocardiographic abnormalities of tricuspid valve motion in 2 patients with left ventricular to right atrial shunts are described. In both patients the abnormal anatomy was defined at surgery, in one patient the shunt being above the tricuspid valve leaflets (supravalvar) and in the other patient through the septal leaflet (intravalvar). Different patterns of tricuspid valve systolic fluttering were seen in these two cases and the possible reasons for this are discussed. After surgical closure of the defects the systolic fluttering of the tricuspid valve was no longer observed. Echocardiography appears to be useful in detecting the presence of left ventricular to right atrial shunts which otherwise may be difficult to diagnose.

The clinical diagnosis of a shunt from the left ventricle to the right atrium (LV-RA) is difficult, because the findings resemble those of an interventricular communication (Gerbode et al., 1958). At cardiac catheterisation, an oxygen saturation step-up at the right atrial level, in the absence of any other features suggesting an atrial septal defect, may be confusing if the diagnosis of an LV-RA shunt has not been considered. A characteristic echocardiographic finding in this condition would, therefore, be of considerable value, not only in detecting the abnormality, but also in the appropriate planning of the diagnostic cardiac catheterisation. The present report concerns 2 patients with LV-RA shunts in whom striking abnormalities of the systolic motion of the tricuspid valve were noted. These findings amplify two previously reported cases (Nanda et al., 1975) and confirm the diagnostic value of echocardiography in this condition.

\section{Patients and methods}

\section{METHODS}

The echocardiographic records were obtained using an Ekoline ultrasonoscope ${ }^{\mathbf{l}}$ interfaced with a Cambridge Physiological Recorder. ${ }^{2}$ Further tracings were obtained in the second patient by interfacing the Ekoline with an Irex Multichannel

\footnotetext{
'Smith-Kline Ekoline 20 Ultrasonoscope. Smith Kline Instruments, Inc., Palo Alto, California, U.S.A.

${ }^{2}$ Cambridge Medical Instruments Co., Ossining, New York, U.S.A.

Received for publication 25 October 1976
}

Recorder. ${ }^{3}$ The recording speed was $100 \mathrm{~mm} / \mathrm{s}$. The echocardiograms of the tricuspid valve were amplified to allow greater appreciation of the detail of valve motion. In each patient a number of echocardiographic scans were recorded to ensure that it was the anterior of two atrioventricular valves that was labelled 'tricuspid'.

\section{PATIENTS}

\section{Case 1}

This 19-year-old girl had a ventricular septal defect diagnosed at the age of 10 . Though surgical closure was recommended, the patient's parents refused operation. She was lost to follow-up but now returned complaining of easy fatiguability and nonspecific chest pain. On physical examination the venous pressure was raised and the right ventricular impulse was prominent.

A loud pansystolic murmur and a low pitched diastolic murmur, interpreted as a diastolic flow murmur, were heard at the left sternal edge. Cardiac catheterisation showed a pulmonary artery pressure of $70 / 25 \mathrm{mmHg}$ and a step-up in oxygen saturation at the right ventricular level. Dye dilution curves indicated shunts from the left ventricle to both the right ventricle and right atrium. A catheter could be readily passed from left ventricle to right atrium.

Case 2

A 33-year-old man who gave a history of a heart murmur since the age of 2 years presented complain'Irex Medical System, Mahwah, New Jersey, U.S.A. 


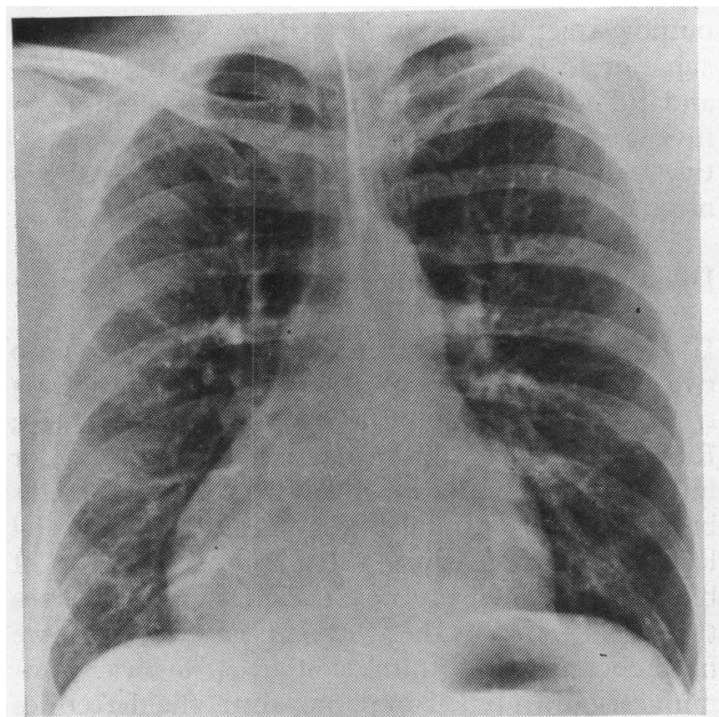

Fig. 1 Posteroanterior chest $x$-ray of case 2, showing distinct prominence of the right heart border resulting from right atrial dilatation.

ing of mild dyspnoea. On examination his venous pressure was not raised, but there was a prominent systolic pulsation to the right of the sternum. A loud pansystolic murmur was audible throughout the praecordium, prominent at both the left and right sternal edge. Chest $x$-ray examination showed striking right atrial enlargement (Fig. 1). Cardiac catheterisation revealed a step up in oxygen saturation at right atrial level. Dye dilution curves indicated a shunt from left ventricle to right atrium, and the left ventricular catheter could be passed directly into the right atrium.

\section{Results}

\section{ECHOCARDIOGRAPHY}

Case 1

The abnormal echocardiographic features of the systolic motion of the tricuspid valve are illustrated in Fig. 2. During ventricular systole the closed tricuspid valve is seen to flutter. This fluttering could be observed throughout the duration of the pansystolic murmur in early systole with the transducer in the fourth intercostal space and in late systole with the transducer in the fifth intercostal space. This abnormal systolic motion was consistently observed when the transducer was angled inferiorly from the aortic valve toward the point of tricuspid valve closure. The amplitude of the fluttering motion is approximately $3.5 \mathrm{~cm}$.

\section{Case 2}

The echocardiographic findings in case 2 also showed an abnormality of tricuspid valve systolic motion and this is illustrated in Fig. 3. One of the tricuspid valve leaflets bows anteriorly into the right

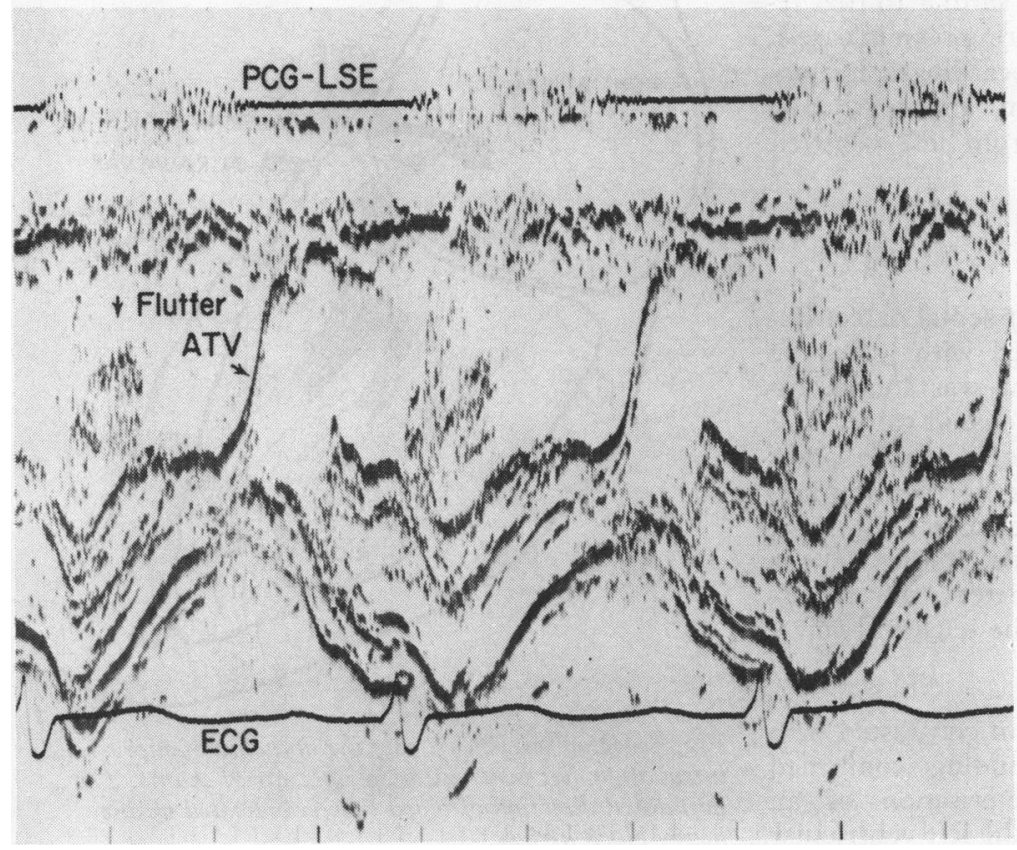

Fig. 2 Echophonocardiogram from case 1. The anterior leaflet of the tricuspid valve ( $A T V$ ) is seen opening at the start of diastole. A fluttering motion of the tricuspid valve is seen in systole. In this view the onset of the pansystolic murmur and the fluttering coincided, and in other tracings the fluttering was shown in late systole. Recording speed $100 \mathrm{~mm} / \mathrm{s}$. Time lines $0.04 \mathrm{~s}$. PCG LSE-phonocardiogram at left sternal edge. 


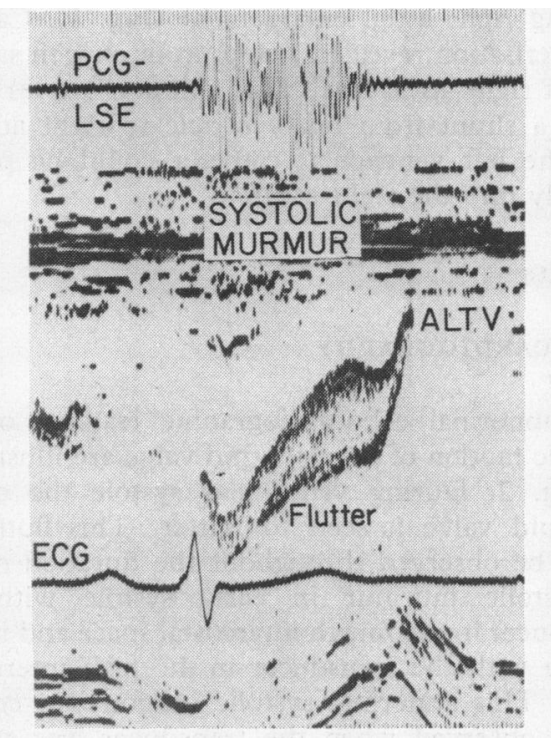

Fig. 3 Echophonocardiogram from case 2 showing the pattern of systolic flutter of the tricuspid valve seen in the patient with a supravalvar left ventricular right atrial shunt. The fluttering coincided with the pansystolic murmur. Recording speed $100 \mathrm{~mm} / \mathrm{s}$. Time lines $0.01 \mathrm{~s}$.

ventricle and a fluttering motion is seen on the leaflet throughout the duration of the pansystolic murmur. This was consistently found on scanning from tricuspid valve closure to the aortic valve echo, the transducer angle being similar to that required to detect the systolic fluttering seen in case 1 .

We have not seen these echocardiographic abnormalities in patients with an atrioventricular canal defect causing a shunt from left ventricle to right atrium.

\section{SURGICAL FINDINGS}

Case 1

A $2.5 \mathrm{~cm}$ membranous ventricular septal defect was identified at operation and closed with a Dacron patch. A separate LV-RA shunt was found, involving the septal leaflet of the tricuspid valve. This communication took the form of multiple small defects in the valvular tissue near its insertion into the atrioventricular ring. These were closed by direct suture. Careful echocardiographic studies in the postoperative period failed to detect any abnormality of the systolic portion of the tricuspid valve.

\section{Case 2}

The surgical findings in this patient contrasted with those in case 1 . The anatomical findings confirmed the diagnosis at cardiac catheterisation of an isolated communication between the left ventricular outflow tract and the right atrium. The defect lay superior to the septal leaflet of the tricuspid valve and did not involve that structure. The communication was repaired by direct suture. The postoperative echocardiogram of the tricuspid valve was normal.

\section{Discussion}

LV-RA shunts have been previously divided into two categories-infravalvar and supravalvar (Perry et al., 1949; Braunwald and Morrow, 1960; Riemenschneider and Moss, 1967). In type 1 or intravalvar, the defect involves both the membranous interventricular septum and the septal leaflet of the tricuspid valve. Since the flow of blood from the left ventricle to right atrium traverses the septal leaflet of the tricuspid valve (Fig. 4), we have chosen to call this lesion an intravalvar communication. In type 2 or supravalvar, the defect lies higher in the interventricular septum so that it is above the level of the insertion of the tricuspid valve (Fig. 4).

The first of the two cases reported here was of the intravalvar type, the second, supravalvar. The 2 previously reported cases of LV-RA shunts with tricuspid valve echocardiography were of the intravalvar type, though the anatomy was verified at

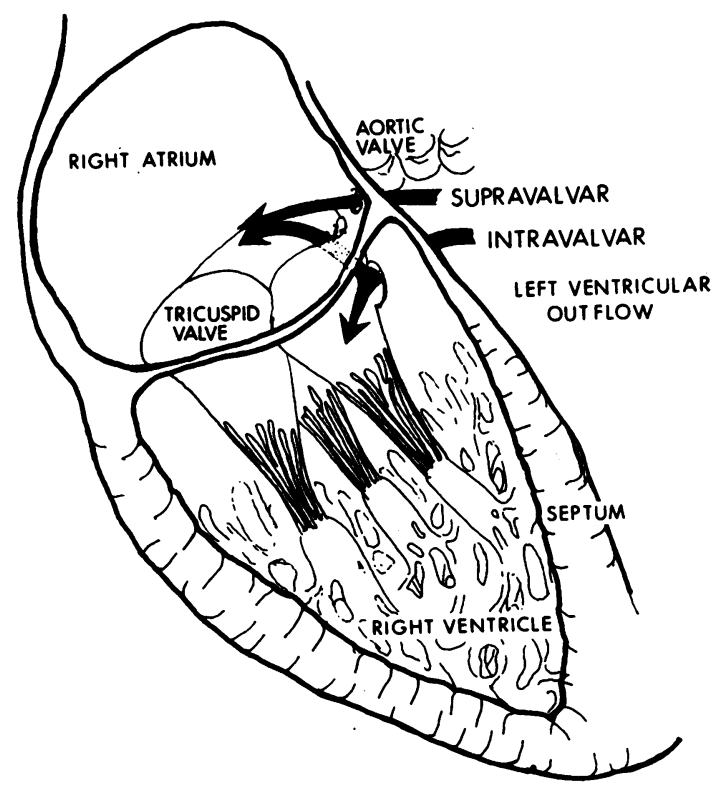

Fig. 4 Diagram illustrating the anatomy of supra-and intravalvar left ventricular to right atrial shunts. The postulated direction of blood flow is indicated by the heavy black arrows. 
surgery in only one of these patients (Nanda et al., 1975). In our patient with this defect (case 1) we found systolic fluttering of the valve, similar to that reported by Nanda et al., though the amplitude of this motion was considerably greater. This increased amplitude may have been the result of the significant pulmonary hypertension present in our patient which would tend to cause right ventricular dilatation and, therefore, improve the echocardiographic recording of the tricuspid valve.

Supravalvar LV-RA shunts are less common than the intravalvar type. The systolic motion of the tricuspid valve in case 2 , which was of a different pattern from that seen in the intravalvar defect, has not previously been reported. The 'flutter' seen with the supravalvar defect was of a higher frequency, being similar in appearance to that seen on the mitral valve in association with aortic regurgitation. The mechanism of tricuspid valve fluttering in LV-RA shunts is conjectural, but the following indirect evidence suggests (1) that the jet of blood impinges on the tricuspid valve in this defect and (2) that the effect of such a jet might be detected echocardiographically.

The patient described by Perry et al. was a child who died from bacterial endocarditis associated with a supravalvular LV-RA shunt. At necropsy vegetations were found on the septal and anterior leaflets of the tricuspid valve, suggesting that during life, the left ventricular jet of blood had impinged directly on the tricuspid leaflets. A recent report (Matsumoto et al., 1976) of systolic fluttering of the tricuspid valve being seen in a patient with ruptured sinus of Valsalva into the right atrium, suggests that the effect of a jet of blood from the left heart on the tricuspid valve during systole may result in the echocardiographic recognition of tricuspid valve flutter. This is a reasonable explanation for the echocardiographic findings in our patient with a supravalvular LV-RA shunt. The different pattern of flutter in the intravalvular shunt is more difficult to explain. The lack of anterior 'bowing' may reflect the fact that during systole motion of the septal leaflet of the tricuspid valve is influenced by blood impinging on both the ventricular and the atrial aspects of the valve cusp. There would thus be no net movement of the cusp into either chamber but rather the pattern of oscillation seen in Fig. 2 would result.

After surgical closure of the defects in both our patients, the echocardiographic abnormalities of systolic motion of the tricuspid valve were no longer present. These findings show that echocardiography of the tricuspid valve may be of considerable value in the detection of left ventricular-right atrial shunts, and suggest that careful echocardiographic study of tricuspid valve motion in patients with the signs of ventricular septal defect would be of value in establishing this diagnosis. The two anatomical variations of this lesion may cause different patterns of flow through or across the tricuspid valve leaflets, hence causing differences in systolic motion of the leaflets and allowing the two variants to be separated echocardiographically.

\section{References}

Braunwald, E., and Morrow, A. G. (1960). Left ventriculoright atrial communication. American fournal of Medicine, 28, 913-920.

Gerbode, F., Hultgren, H., Melrose, D., and Osborn, J. (1958). Syndrome of left ventricular-right atrial shunt. Annals of Surgery, 148, 433-446.

Matsumoto, M., Matsuo, H., Beppu, S., Yoshioka, Y., Kawashima, Y., Nimura, Y., and Abe, H. (1976). Echocardiographic diagnosis of ruptured aneurysm of sinus of Valsalva. Circulation, 53, 382-389.

Nanda, N. C., Gramiak, R., and Manning, J. A. (1975). Echocardiography of the tricuspid valve in congenital left ventricular-right atrial communication. Circulation, 51, 268-272.

Perry, E. L., Burchell, H. B., and Edwards, J. E. (1949). Cardiac clinics; congenital communication between the left ventricle and the right atrium: coexisting ventricular septal defect and double tricuspid orifice. Proceedings of the Staff Meetings of the Mayo Clinic, 24, 198-206.

Riemenschneider, T. A., and Moss, A. J. (1967). Left ventricular-right atrial communication. American fournal of Cardiology, 19, 710-718.

Requests for reprints to Dr. Ernest Craige, Department of Medicine, 338 Clinical Sciences Building 229H, UNC School of Medicine, Chapel Hill, North Carolina, U.S.A. 